\title{
The acquisition of three types of auditory pattern discrimination in the cat*
}

\author{
JACK B. KELLY \\ Carleton University, Ottawa, Ontario, Canada K1S $5 B 6$
}

\begin{abstract}
This study compared the learning ability of cats in shock-avoidance discrimination with temporally patterned sounds. The three discriminations used involved detection of rising vs falling frequency-modulated tones, low-high vs high-low pairs of pure tones, and rising-falling vs falling-rising pairs of frequency-modulated tones. The discrimination of rising vs falling frequency-modulated tones was more easily learned than the other two discriminations.
\end{abstract}

Although temporal patterns constitute an important part of the auditory environment, relatively little attention has been paid to the ability of animals to discriminate patterned auditory stimuli. Temporal patterns composed of pure tones have been used with monkeys and cats (Jerison \& Neff, 1953; Diamond \& Neff, 1957; Elliott \& Trahiotis, 1972) but have proved to be relatively difficult discriminations to learn (Cowey, 1968). On the other hand, Kelly and Whitfield (1971) have used temporal patterns composed of frequency-modulated tones and have found that cats easily learn to discriminate rising from falling tones. These results suggest a basic difference between FM patterns and pure tone patterns, although comparison is complicated by differences in intensity, duration, and frequency range of different patterns. In the following study, a direct comparison is made among three pattern discriminations involving frequency-modulated tones and pure-tone bursts.

\section{METHODS}

Twelve male adult cats were involved in pattern-d iscrimination tests. Each of the animals was first trained in a double-grill box to make an avoidance response to the onset of a buzzer. In this, and in subsequent tasks, a daily training session consisted of five warning trials presented at random intervals between 60 and $300 \mathrm{sec}$. Each buzzer-warning trial was $10 \mathrm{sec}$ long, and the cats were trained to cross during this period from one side of the box to the other to avoid a shock. When a cat reached a criterion of 9 out of 10 correct responses over 2 days, training with patterned stimuli was begun.

Three different pattern discriminations were employed. Four cats were assigned to each of the discriminations. With the exception of the stimulus configuration, the training procedure was the same for the three discriminations. A patterned safe signal was presented in the background at a rate of $1 / \mathrm{sec}$. During a training trial, the safe signal was replaced by a warning signal. The cats were expected to respond to this signal within $10 \mathrm{sec}$ in order to avoid a shock. In addition to the number of correct responses, the number of spontaneous responses was estimated from the number of $10-\mathrm{sec}$ periods between trials during which a response occurred. A discrimination score for each session was

*The author would like to thank the National Research Council of Canada for their support.

based on the difference between percentages of correct responses and spontaneous responses.

The differences of the three temporal pattern discriminations are illustrated in Fig. 1. In the first discrimination (FM), the safe signal consisted of a series of rising frequency-modulated tones and the warning signal was a series of falling frequency-modulated tones. The range of frequencies covered was between $3,780 \mathrm{~Hz}$ and $4,220 \mathrm{~Hz}$. In the second discrimination (TONE PAIRS), the safe signal was a series of low-high tone pairs. The warning signal was a series of high-low pairs. The two tones were $4,220 \mathrm{~Hz}$ and $3,780 \mathrm{~Hz}$, separated by an 80 -msec silent interval. In the third discrimination (FM PAIRS), the safe signal was a series of paired frequency-modulated tones, rising then falling. The rising component was separated from the falling componenet by $80 \mathrm{msec}$. The warning signal was a series of paired frequency-modulated tones in which the falling component preceded the rising component. The terminal frequencies of these modulated tones were $4,220 \mathrm{~Hz}$ and $3,780 \mathrm{~Hz}$. As can be seen in Fig. 1, the total duration of each stimulus configuration

FM

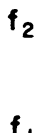

$f_{2}$

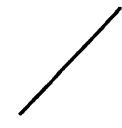

vs

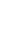

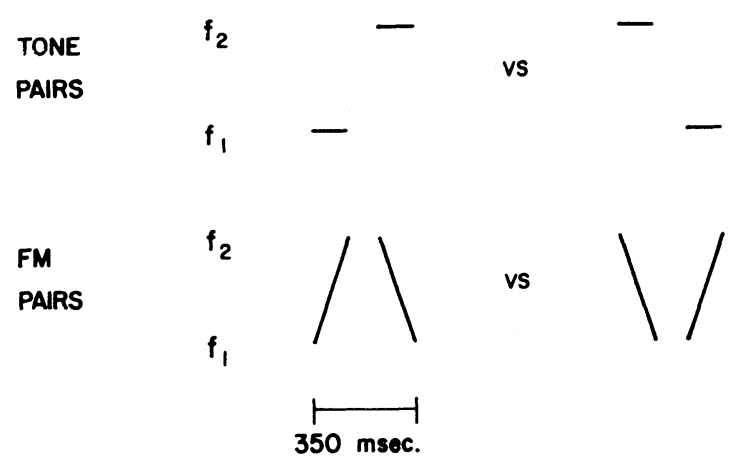

Fig. 1. Illustration of the three discriminations used. The stimulus configuration for the safe signal is shown on the left. The warning signal is shown on the right. Notice that the frequency excursion from $f_{1}$ to $f_{2}$ is the same for each configuration, i.e., 3,780 to $4,220 \mathrm{~Hz}$. Also, the duration, $350 \mathrm{msec}$, is the same for each stimulus. (Onset and of fset times were $15 \mathrm{msec}$ for all stimulus presentations.) 
was $350 \mathrm{msec}$. The sound pressure level was the same for each as was the rate of presentation $(1 / \mathrm{sec})$. The safe signal and warning signal for each discrimination were identical in frequency content and therefore could be distinguished only on the basis of temporal order.

\section{RESULTS}

The ease of learning the three pattern discriminations was not the same. The four cats trained to discriminate rising from falling FM patterns reached a criterion of 9 out of 10 correct responses over 2 days in $11,16,18$, and 19 sessions. None of the animals on the other two pattern discriminations reached this criterion. Training was continued for 20 sessions with pure-tone pairs and 15 sessions for FM pairs and, during these sessions, performance remained at a very low level. The level of spontaneous responses was generally quite low for each of the three discriminations. Means were $6.8 \%$ for the FM task, $7.0 \%$ for the tone pairs, and $9.7 \%$ for FM pairs. Therefore, differences in performance were not due simply to large differences in spontaneous activity. Performance levels for each of these discriminations are shown as average discrimination scores in Fig. 2. The FM pattern discrimination was more rapidly acquired than the other two discriminations. Statistical analysis of performance levels was carried out separately for sessions 1-5, 6-10, and 11-15 using the Kruskal-Wallis test (Siegel, 1956). Differences in performance were statistically significant $(p<.05)$ for the second and third five-trial blocks.

The inability of the animals trained on patterns of tone pairs and FM pairs to perform these discriminations does not mean that the discriminations are impossible for cats. Indeed, the four animals trained originally to discriminate rising from falling tones were able to learn the discrimination of low-high vs high-low tone pairs in an average of 10 additional sessions. Furthermore, the four cats which failed initially to learn the discrimination of tone pairs did learn to discriminate rising from falling FM tones in an average of 8.25 sessions and were subsequently able to discriminate low-high pairs in an average of 8.50 sessions.

\section{DISCUSSION}

Rising vs falling frequency-modulated patterns are more easily leamed by cats than are patterns of tone pairs or FM pairs, even when the procedures used in training the cats are the same and the methods of stimulus presentation, the intensity, frequency range, duration, and repetition rate are identical. The difference in difficulty under these testing conditions must, therefore, be related to the temporal configuration of the stimuli. Also, the ease of learning the FM pattern task is not due simply to the use of frequency-modulated tones, although the complexity of such stimuli and their relevance to environmental sounds or the animal's past experience are possible factors. Pattern discriminations with paired FM tones are not so easily learned.

Kelly and Whitfield (1971) have suggested that the ease of learning to discriminate rising from falling FM sounds is related to the way in which these stimuli are processed by the central nervous system. Neurons at several levels of the auditory pathway are sensitive to directional properties of frequency-modulated stimuli (Erulkar, Butler, \& Gerstein, 1968; Fernald \& Gerstein, 1972; Nelson, Erulkar \& Bryan, 1966; Whitfield \& Evans, 1965). Indeed, at the level of the auditory cortex, some cells respond vigorously to modulation in one direction but not at all to modulation in the other direction (Whitfield \& Evans, 1965). Thus, within the central nervous system, a somewhat different population of cells will respond to rising and falling frequency-modulated tones, even though the same frequency range is covered by both stimuli. This type of response suggests an interaction between the elements excited by different frequency components of the stimulus (Erulkar, Nelson, \& Bryan, 1968; Whitfield, 1969). The opportunity for this kind of interaction

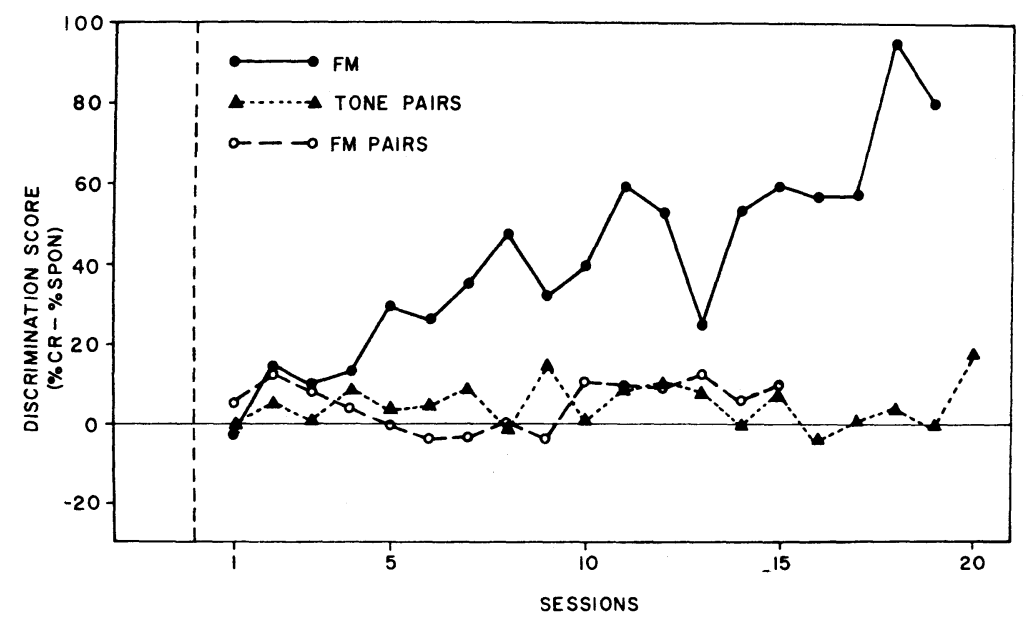

Fig. 2. The average discrimination scores for animals tested under the three stimulus conditions. Solid circles represent FM patterns, solid triangles represent tone pairs and open circles represent FM pairs. Because training was discontinued when an animal reached criterion, the later sessions for FM patterns represent fewer than the normal four cases per group. 
would be less when a temporal gap is introduced between the elements which make up a pattern. In the case of paired pure tones, low-high vs high-low, a temporal gap of $80 \mathrm{msec}$ separates the two frequency components. Similarly, with paired FM patterns a temporal gap exists between the rising and falling components of the stimulus configuration. In both these pattern discriminations, there is a different requirement for temporal integration than in the case of rising and falling FM stimuli.

The suggestion that differences in central processing exist for FM and pure-tone patterns is confirmed by studies of the effect of central nervous system lesions. In behavioral-discrimination tasks with cats, auditory cortical lesions result in severe impairments in discriminations of pure-tone patterns (Diamond \& Neff, 1957; Goldberg, Diamond, \& Neff, 1957). Comparable lesions have a much less pronounced effect in discriminations of rising and falling FM patterns (Kelly $\&$ Whitfield, 1971). Following insular-temporal lesions, cats can learn discriminations of rising vs falling FM patterns, but fail to learn low-high vs high-low pure-tone patterns (Kelly, 1973). Thus, differences exist in the cortical dependency of these two types of discrimination. Differences in central processing have also been proposed by Nabelek et al (1973) to explain perceptual features of continuous and discontinuous frequency changes in humans.

\section{SUMMARY}

Twelve cats were trained in a shock-avoidance task to discriminate temporally patterned sounds. The discriminations used were rising vs falling frequency-modulated tones, low-high vs high-low pairs of pure tones, and rising-falling vs falling-rising pairs of frequency-modulated tones. Comparison of the rate of acquisition of animals on these discriminations showed that rising vs falling FM pattern was more easily learned than the other two discriminations.

\section{REFERENCES}

Cowey, A. Discrimination. In L. Weiskrantz (Ed.), A nalysis of behavioral change. New York: Harper \& Row, 1968.

Diamond, I. T., \& Neff, W. D. Ablation of temporal cortex and discrimination of auditory patterns. Journal of Neurophysiology, 1957, 20, 300-315.

Elliott, D. N., \& Trahiotis, C. Cortical lesions and auditory discrimination. Psychological Bulletin, 1972, 77, 198-222.

Erulkar, S. D., Butler, R. A., \& Gerstein, C. L. Excitation and inhibition in cochlear nucleus. II. Frequency-modulated tones. Journal of Neurophysiology. 1968, 31, 537-548.

Erulkar, S. D., Nelson, P. G., \& Bryan, J. S. Experimental and theoretical approaches to neural processing in the central auditory pathway. In W. D. Neff (Ed.), Contributions to sensory physiology Vol. 3. New York: Academic Press, 1968. sensory physion.

Fernald, R. D., \& Gerstein, C. L. Response of cat cochlear nucleus neurons to frequency and amplitude modulated tones. Brain Research, 1972, 45, 417-435.

Goldberg, J. M. Diamond, I. T, \& Neff, W. D. Auditory discrimination after ablation of temporal and insular cortex in the cat. Federation Proceedings, 1957, 16, 47.

Jerison, H. J., \& Neff, W. D. Effects of cortical ablation in the monkey and discrimination of auditory patterns. Federation Proceedings, 1953, 12, 73-74.

Kelly, J. B. The effects of insular and temporal lesions in cats on two types of auditory pattern discrimination. Brain $R$ esearch, $1973,62,71-87$.

Kelly, J. B., \& Whitfield, I. C. Effects of auditory cortical lesions on discriminations of rising and falling frequency modulated tones. Journal of Neurophysiology, 1971, 34, 802-816.

Nabelek, I. V., Nabelek, A. K., \& Hirsch, I. J. Pitch of sound bursts with continuous and discontinuous change of frequency. Journal of the Acoustical Society of America, $1973,53,1305-1312$.

Nelson, P. G., Erulkar, S. D., \& Bryan, J. S. R esponses of units of the inferior colliculus to time-varying acoustic stimuli. Journal of Neurophysiology, 1966, 29, 834-860.

Siegel, S. Nonparametric statistics for the behavioral sciences. New York: McGraw-Hill, 1956.

Whitfield, I. C. R esponses of the auditory nervous system to simple time-dependent acoustic stimuli. Annals of the New York Academy of Sciences, 1969, 156, 671-677.

Whitfield, I. C., \& Evans, E. F. R esponses of auditory cortical neurons to stimuli of changing frequency. Journal of Neurophysiology, 1965, 28, 655-672.

(Received for publication February 21, 1974; accepted July 23, 1974.) 